\title{
A Tunable 3-Terminal GMR Device Based on a Hybrid Magnetic-Electric-Barrier Nanostructure
}

\author{
Y. H. Kong, S. Y. Chen, G. L. Zhang, and X. Fu \\ Department of Electron Engineering, Hunan University of Science and Engineering, Yongzhou, Hunan 425100, China \\ Correspondence should be addressed to Y. H. Kong; y_h_kong@163.com
}

Received 27 July 2012; Accepted 12 October 2012

Academic Editor: Mao-Wang Lu

Copyright (C) 2013 Y. H. Kong et al. This is an open access article distributed under the Creative Commons Attribution License, which permits unrestricted use, distribution, and reproduction in any medium, provided the original work is properly cited.

\begin{abstract}
We propose a giant magnetoresistance (GMR) device, which can be experimentally realized by depositing two ferromagnetic (FM) strips and a Schottky metal (SM) stripe in parallel configuration on top of the GaAs heterostructure. The GMR effect ascribes a significant electron transmission difference between the parallel and antiparallel magnetization configurations of two FM stripes. Moreover, the MR ratio depends strongly on the magnetic strength of the magnetic barrier (MB) and the electric barrier (EB) height induced by an applied voltage to the SM stripe. Thus, this system can be used as a GMR device with tunable MR by an applied voltage to SM stripe or by magnetic strength of the MB.
\end{abstract}

\section{Introduction}

Since the discovery of the giant magnetoresistance (GMR) effect in 1988 [1], GMR systems have attracted a wealth of experimental and theoretical attentions in recent years. GMR effect has given rise to a huge impact on the magnetic information storage $[2,3]$, such as ultrasensitive magnetic field sensors, read heads, and random access memories [4,5]. In general, the GMR effect is observed in a sandwiched structure composed of two ferromagnetic (FM) layers separated by a thin nonmagnetic layer, when the relative orientations of the magnetizations of these two FM layers are switched, by an externally applied magnetic field, from an antiparallel (AP) to a parallel (P) alignments. For the $\mathrm{P}$ configuration, the conductance of the structure is highest since the spindependent scattering of the carriers is minimized, while the conductance is lowest for the AP configuration due to the maximized spin-dependent carrier scattering. In a GMR device, the current can either be perpendicular to the planes of the layers (i.e., the so-called CPP geometry) or be parallel to the interfaces (the CIP geometry). Moreover, the degree of the GMR effect is usually characterized by a striking drop of the electric resistance from $\mathrm{P}$ to AP alignment, that is, the magnetoresistance (MR) ratio that is defined by $\mathrm{MR}=$ $\left(G_{\mathrm{P}}-G_{\mathrm{AP}}\right) / G_{\mathrm{AP}}$, where $G_{\mathrm{P}}$ and $G_{\mathrm{AP}}$ are the conductances for
P and AP alignments, respectively. For a specific GMR device, from the viewpoint of practical applications, it is desirable for a high MR under relatively low saturation magnetic field.

To obtain a large MR ratio, recent studies [6-9] demonstrate that an attractive way is to exploit the so-called magnetic barrier (MB) nanostructure [10]. Experimentally, the $\mathrm{MB}$ nanostructure can be fabricated $[11,12]$ by deposing a nanosized FM material on top of a near-surface twodimensional electron gas (2DEG) formed in a modulationdoped semiconductor heterostructure, where the FM material provides a magnetic field which can influence locally the motion of the electrons in the semiconductor heterostructure. Several groups [6-9] have explored the GMR effect of $\mathrm{MB}$ nanostructures. It has been found that, although the average magnetic field of the structure is zero, this kind of system possesses very high MR ratio, and the GMR effect makes no use of the spin degree of freedom distinct from the conventional GMR devices.

Recently, based on the combination of FM stripe, Schottky-metal (SM) stripe and semiconductor heterostructure, Zhai et al. [13] proposed a novel electron-spin filter. They found that, an intrinsic symmetry in the single FM stripe $\mathrm{MB}$ nanostructure can be broken due to the inclusion of SM stripe, which results in an evident electron-spin filtering effect. Nevertheless, this conclusion is reasonable only for 
the InAs material system [14]. Considering that the MB nanostructure usually is fabricated on the conventional $\mathrm{GaAs}$ heterostructure [10-12], in the present work we will ignore the spin effect on electronic transport properties in the novel device proposed by Zhai et al. Although much current attention has been paid to the study of the GMR effect for the $\mathrm{MB}$ nanostructures, to the best of our knowledge, at the moment the study of the GMR effect in the above spin-filter is still an open question.

\section{Proposed Device Structure}

The main purpose of this paper is to study the GMR effect of the device, as depicted in Figure 1(a). This device consists of a 2DEG in the $(x, y)$ plane subject to modulations by two FM stripes and an SM stripe, where the width of the stripe is $d, D$ is the spacing between FM and SM stripes, and $z_{0}$ the distance between stripes and the 2DEG. Two FM stripes are assumed to be magnetized along the $x$-axis in Figure $1(\mathrm{a})$, and the magnetic fields provided by the FM stripes $B_{z}(x)$ can be approximated $[7,8]$ as $\delta$-function, in order to demonstrate the principle of operation of this device. Here, $B$ is the magnetic strength of $\delta$-function barrier, $U(x)$ stands for the electric potential induced by the applied voltage to the SM stripe, and Figures 1(b) and 1(c) correspond to the parallel (P) and antiparallel (AP) configurations of two FM layers, respectively. Assume that the magnetic field $B_{z}(x)$ and the electrical potential $U(x)$ are homogeneous in the $y$ direction and vary only along the $x$-axis.

\section{Theoretical Analysis}

The motion of an electron in such a modulated 2DEG system can be described by the single-particle Hamiltonian,

$$
H=\frac{p_{x}^{2}}{2 m^{*}}+\frac{\left[p_{y}+e A_{y}(x)\right]^{2}}{2 m^{*}}+U(x)
$$

where $m^{*}$ is the effective mass, $\left(p_{x}, p_{y}\right)$ is the electron momentum, and $\mathbf{A}(x)=A_{y}(x) \hat{e}_{y}$ is the magnetic vector potential under the Landau gauge. For convenience we express all quantities in dimensionless units by means of two characteristic parameters, that is, cyclotron frequency $\omega_{c}=e B_{0} / m^{*}$ and magnetic length $\ell_{B}^{2}=\hbar / e B_{0}\left(B_{0}\right.$ being some typical magnetic field). In our numerical calculations, the GaAs system will be taken as the 2DEG material $\left(m^{*}=\right.$ $0.067 m_{0}$ with $m_{0}$ being the mass of free electron in vacuum), thus the reduced units are $\ell_{B}=81.3 \mathrm{~nm}$ and $E_{0}=\hbar \omega_{c}=$ $0.17 \mathrm{meV}$, corresponding to $B_{0}=0.1 \mathrm{~T}$.

Because of the translational invariance of the system along the $y$ direction, the total electronic wave-function can be written as $\Phi(x, y)=e^{i k_{y} y} \psi(x)$, where $k_{y}$ is the longitudinal wave vector, and $\psi(x)$ satisfies the reduced one-dimensional (1D) Schrodinger equation:

$$
\left\{\frac{d^{2}}{d x^{2}}-\left[k_{y}+A_{y}(x)\right]^{2}+2[E-U(x)]\right\} \psi(x)=0 .
$$

It is useful to introduce the effective potential of the nanostructure:

$$
U_{\mathrm{eff}}\left(x, k_{y}\right)=\frac{\left[k_{y}+A_{y}(x)\right]^{2}}{2}+U(x) .
$$

Clearly, this effective potential of the nanostructure depends strongly not only on the magnetic configuration $B_{z}(x)$ via the vector potential $A_{y}(x)$, but also on the longitudinal wave vector $k_{y}$. The $k_{y}$-dependence renders the motion of electrons an essentially two-dimensional (2D) process as would be expected from the classical analogy. From the dependence of the $U_{\text {eff }}$ on the magnetic profile $B_{z}(x)$, one can easily see that, for the device presented in (Figure 1(a)), when the P alignment (Figure 1(b)) turns to the inverse Figure 1(c), $U_{\text {eff }}$ varies substantially. It is the dependence on the magnetic configuration of $U_{\text {eff }}$ that results in the GMR effect in the nanosystem.

Matching the wave functions and their derivatives at interfaces, the transmission probability through this nanostructure $T\left(E, k_{y}\right)$ for electrons with incident energy $E$ and wave vector $k_{y}$ can be determined by the transfer matrix method [15]. Then, we can calculate the ballistic conductance at zero temperature from the well-known Landauer-Buttiker formula [16]:

$$
G\left(E_{F}\right)=G_{0} \int_{-\pi / 2}^{\pi / 2} T\left(E_{F}, \sqrt{2 E_{F}} \sin \vartheta\right) \cos \vartheta d \vartheta
$$

with $\vartheta$ the incident angle relative to the $x$ direction. The conductance is presented in units of $G_{0}=2 e^{2} m^{*} v_{F} L_{y} / h^{2}$, where $v_{F}$ is the Fermi velocity and $L_{y}$ is the longitudinal length of the system.

\section{Results and Discussion}

To begin with, in order to exhibit the discrepancy of transmissions for electrons tunneling through $\mathrm{P}$ and $\mathrm{AP}$ configurations (Figures 1(b) and 1(c)), we have calculated the corresponding transmission probabilities, that is, $T_{\mathrm{P}}$ and $T_{\text {AP }}$. Figure 2 gives the transmission probability versus the incident energy $E$ for electrons with different wave vector $k_{y}=0$ (solid curve), 2 (dashed curve), and -2 (dotted curve), where Figures 2(a) and 2(b) correspond to the $\mathrm{P}$ and AP alignments, respectively. The structural parameters are chosen to be $B=5, U=4, d=1$, and $D=1$ for both alignments. For the $\mathrm{P}$ configuration, we can see clearly from the Figure 2(a) that the electronic transmission spectrum exhibits a longitudinal-wave-vector dependent tunneling features as confirmed previously [10], which is due to the essentially $2 \mathrm{D}$ process for electrons through the $\mathrm{MB}$ nanostructure. Furthermore, one can obviously see that in the low-energy region there are several resonant peaks with unity values. This can be expected because for the considered wave vectors $k_{y}$ the effective potential $U_{\text {eff }}$ has a symmetric multiple barrier structure for the P configuration, where the process of electron motion is resonantly tunneling through these barriers. However, when the system switches from the $\mathrm{P}$ configuration to the AP configuration, one can see from 


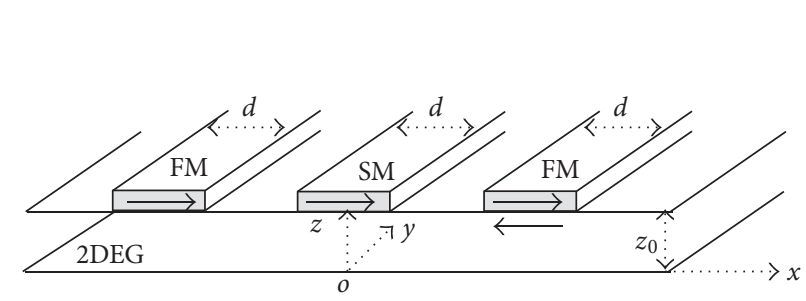

(a)

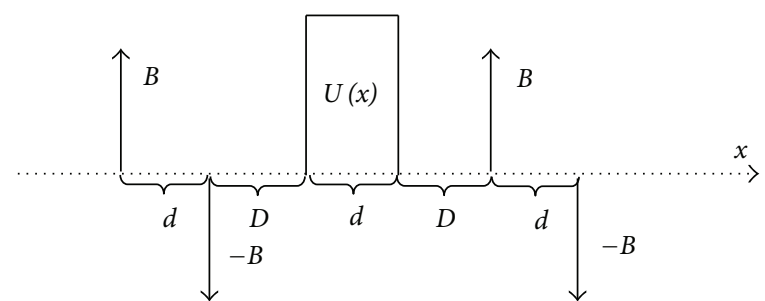

(b)

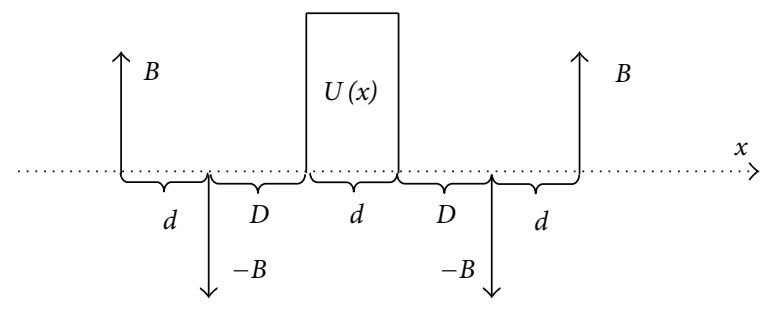

(c)

Figure 1: (a) The schematic illustration of the GMR device, where two FM stripes and one SM stripe are placed on the top of the GaAs heterostructure. (b) and (c) correspond to the P and AP alignments of the device, respectively.
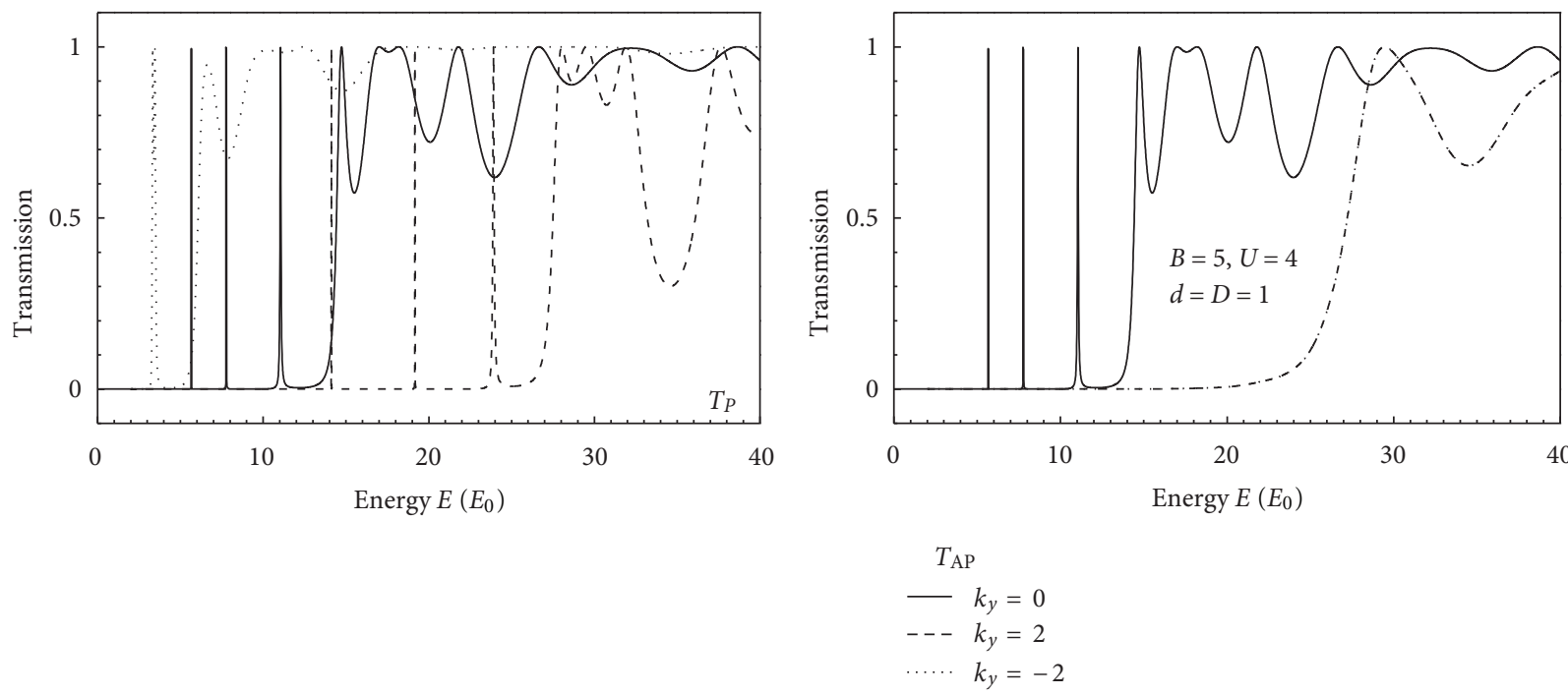

(a)

(b)

Figure 2: (a) and (b) are the transmission coefficients for electrons tunneling through the P and AP configurations, respectively, where the structural parameters are chosen to be $B=5, U=4, d=D=1$, and $D_{1}=D_{2}=1$, as well as the transverse wave vector of electrons is taken to be $k_{y}=0$ (solid curve), 2 (dashed curve), and -2 (dotted curve).

Figure 2(b) that the electron transmission is greatly altered because of the variation of the $U_{\text {eff }}$ induced by the structure. There are several prominent features in $T_{\mathrm{AP}}$ transmission spectrum, which we would like to summarize as follows. (1) In the case of normal incidence (i.e., $k_{y}=0$ ), the transmission coefficient for the AP alignment is exactly the same as that for the $\mathrm{P}$ alignment, because their effective potentials $U_{\text {eff }}$ are identical. (2) The transmission coefficient of electrons with longitudinal wave vector $k_{y}$ is equal to that with $-k_{y}$, that is, in Figure 2(b) the dashed curve superposes the dotted curve. For the AP alignment, the magnetic profile $B_{z}(x)$ and the corresponding vector potential $A_{y}(x)$ are symmetric and antisymmetric with respect to the $x$-axis, respectively, which results in the effective potential $U_{\text {eff }}\left(x, k_{y}\right)=U_{\text {eff }}\left(x,-k_{y}\right)$ according to the well-known fact that for particles traversing a potential in opposite directions the transmission probability is always equal. Therefore, such a symmetry leads to the invariance of the transmission probability with respect to the replacement $k_{y} \rightarrow-k_{y}$, that is, $T_{\mathrm{AP}}\left(E, k_{y}\right)=T_{\mathrm{AP}}\left(E,-k_{y}\right)$. (3) When $k_{y} \neq 0$, the transmission coefficient is strongly suppressed for electrons tunneling through the AP configuration. This is because for the AP alignment the effective potential $U_{\text {eff }}$ is nonsymmetric electric multiple-barriers or wells, in which the electronic transmission is incomplete. 


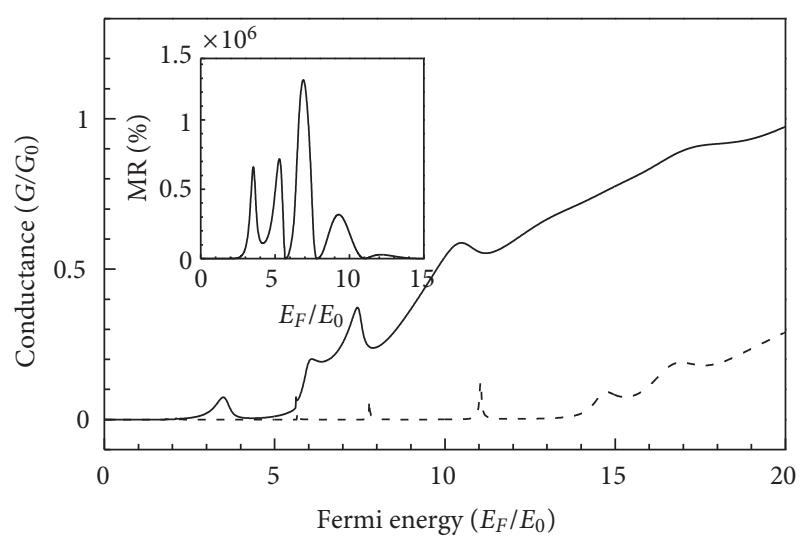

$-G_{\mathrm{AP}}$
$--G_{\mathrm{P}}$

FIGURE 3: The conductances $G_{\mathrm{P}}$ (solid curve) and $G_{\mathrm{AP}}$ (dashed curve) for the $\mathrm{P}$ and AP alignments of the device, where the structural parameters are the same as in Figure 2. Its inset gives the $M R$ ratio MR versus the Fermi energy $E_{F}$.

The configuration-dependent transmission features demonstrated above should be reflected in the measurable quantity, the conductance $G$, which is obtained by integrating the transmission of electrons over the incident angle as in (3). Indeed, our numerical calculation also confirms this discrepancy in the conductance $G_{\mathrm{P}}$ and $G_{\mathrm{AP}}$. In Figure 3 we present the conductance $G_{\mathrm{P}}$ (dashed curve) and $G_{\mathrm{AP}}$ (dotted curve) for the P and AP alignments versus the Fermi energy $E_{F}$, where the structural parameters are the same as in Figure 2 and the conductance is in units of $G_{0}$. The large suppression of the conductance $G_{\mathrm{AP}}$ is clearly seen due to the great reduction of the transmission coefficient $T_{\mathrm{AP}}$ in contrast to the $\mathrm{P}$ alignment. It is this large suppression on the conductance of the AP alignment that results in an evident GMR effect in considered device. The inset of Figure 3 shows that the MR ratio MR as a function of the Fermi energy $E_{F}$ for our considered nanostructure. It is obvious that the MR can be up to $10^{6} \%$ at certain Fermi energy and varies drastically with the Fermi energy $E_{F}$, (namely, it exhibits evident oscillation with respect to the Fermi energy). It is also evident that the GMR effect mainly occurs in the low Fermienergy region and the MR reduces to zero for the large $E_{F}$.

A controlled MR ratio is desirable for practical GMRdevice applications. Now, we examine the influence of the intensity of $\delta$-function $\mathrm{MB}$ and the electric barrier height on the GMR effect for the device presented in Figure 1(a). Firstly, in Figure 4 we show the MR ratio MR as the function of the Fermi energy $E_{F}$ for the different magnetic strength $B=1$ (solid curve), 2 (dashed curve), and 3 (dotted curve), where other structural parameters are the same as in Figure 3. These three MR curves indicate clearly that the MR ratio of the device is sensitive to the intensity of magnetic field $B$. When the magnetic strength $B$ increases, the MR curve shifts towards low Fermi-energy region and its magnitude is uplifted drastically. This feature results from the great

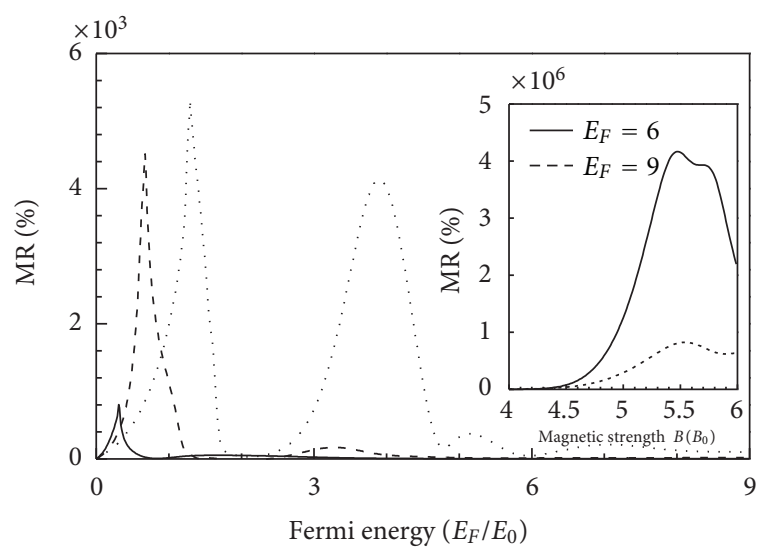

$$
\begin{aligned}
-B & =1 \\
---B & =2 \\
\cdots \cdots & B=3
\end{aligned}
$$

FIgURE 4: The MR ratio MR as a function of the Fermi energy $E_{F}$ for three different magnetic intensities of $\delta$-function MB: $B=1$ (solid curve), 2 (dashed curve), and 3 (dotted curve), where other structural parameters are the same as in Figure 2. The inset shows that the MR as the function of the $B$ for two fixed Fermi energies $E_{F}=6$ (solid curve) and 9 (dashed curve).

variation of effective potential of nanostructure $U_{\text {eff }}$ with the magnetic intensity $B$ of $\delta$-function MB in system, which implies that one can conveniently tune the degree of the GMR effect via adjusting the parameter $B$. To see more clearly the influence of the magnetic field $B$, in the inset of Figure 4 two MR curves calculated as the function of the $B$ are plotted for two fixed Fermi-energy $E_{F}=6$ (solid curve) and 9 (dashed curve). A strong dependence of MR ratio of the device on the magnetic strength of $\delta$-function MB can be seen obviously.

Finally, Figure 5 presents the change of the MR ratio with the Fermi energy at three electric-barrier (EB) height $U=4$ (solid curve), 6 (dashed curve) and 8 (dotted curve). Here, other structural parameters are the same as in Figure 3. From this figure, one can observe evidently that the EB height can alter greatly the MR ratio of the device due to its effective potential $U_{\text {eff }}$ relative closely to the electric potential $U(x)$. The MR curve shifts ratherish towards high Fermienergy direction and its value diminishes obviously as the EB height $U$ increases. In order to demonstrate better the variation of the MR ratio with the $\mathrm{EB}$ height, in the inset of Figure 5 we plot two MR curves as the function of the $U$ for two fixed Fermi-energy $E_{F}=6$ (solid curve) and 9 (dashed curve). Indeed, these two MR curves show that the MR changes greatly its value with altering the EB height $U$. This dependence of the MR ratio on the EB height tells us that the GMR effect of the nanostructure also can be manipulated by changing EB height. Due to the EB height induced by an applied voltage to the SM stripe of the device, one can tune readily the MR ratio by adjusting this applied voltage, which may lead to a voltage-tunable GMR device. 


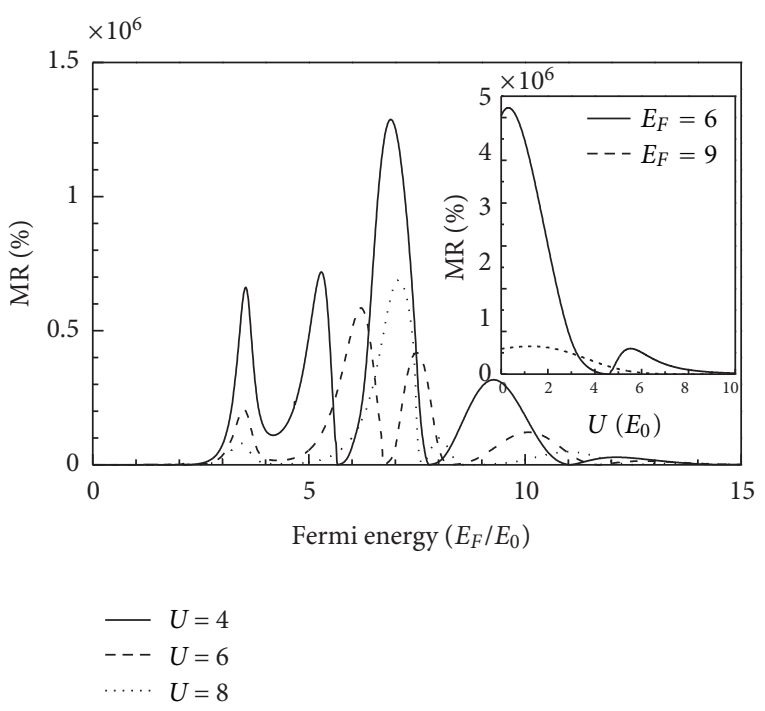

FIgURE 5: The MR ratio MR versus Fermi energy $E_{F}$ for three different EB height $U=4$ (solid curve), 6 (dashed curve), and 8 (dotted curve), where other structural parameters are the same as in Figure 4. The inset gives the MR as the function of the $U$ at two fixed Fermi energies $E_{F}=6$ (solid curve) and 9 (dashed curve).

\section{Conclusions}

In summary, we theoretically investigate the GMR effect in a novel device proposed recently by Zhai et al., which can be experimentally realized by the depositing two FM stripes and one SM stripe on the top of a conventional GaAs heterostructure. Our numerical calculations show that, this device shows up a considerable GMR effect because of the significant electron transmission difference between the $\mathrm{P}$ and AP configurations of device, especially the transmission suppression for the AP alignment. We also have exhibited that the MR ratio is greatly influenced by the magnetic intensity of the $\delta$-function MB and by the EB height (due to an applied voltage to the SM stripe), which may result in a tunable GMR device that its MR ratio is tunable by adjusting the magnetic strength of $\delta$-function $\mathrm{MB}$ or by changing the applied voltage under the SM stripe.

\section{References}

[1] M. N. Baibich, J. M. Broto, A. Fert et al., "Giant magnetoresistance of (001)Fe/(001)Cr magnetic superlattices," Physical Review Letters, vol. 61, no. 21, pp. 2472-2475, 1988.

[2] S. A. Wolf, D. D. Awschalom, R. A. Buhrman et al., "Spintronics: a spin-based electronics vision for the future," Science, vol. 294, no. 5546, pp. 1488-1495, 2001.

[3] C. A. Ross, "Patterned magnetic recording media," Annual Review of Materials Science, vol. 31, pp. 203-235, 2001.

[4] J. M. Daughton, A. V. Pohm, R. T. Fayfield, and C. H. Smith, "Applications of spin dependent transport materials," Journal of Physics D, vol. 32, no. 22, pp. R169-R177, 1999.

[5] J. M. Daughton, "GMR applications," Journal of Magnetism and Magnetic Materials, vol. 192, no. 2, pp. 334-342, 1999.
[6] B.-X. Li, P.-L. Cao, R. Q. Zhang, and S. T. Lee, "Electronic and geometric structure of thin stable short silicon nanowires," Physical Review B, vol. 65, no. 12, Article ID 125305, 2002.

[7] G. Papp and F. M. Peeters, "Giant magnetoresistance in a two-dimensional electron gas modulated by magnetic barriers," Journal of Physics Condensed Matter, vol. 16, no. 46, pp. 8275-8283, 2004.

[8] G. Papp and F. M. Peeters, "Tunable giant magnetoresistance with magnetic barriers," Journal of Applied Physics, vol. 100, no. 4, Article ID 043707, 2006.

[9] X. D. Yang, R. Z. Wang, Y. Guo et al., "Giant magnetoresistance effect of two-dimensional electron gas systems in a periodically modulated magnetic field," Physical Review B, vol. 70, no. 11, Article ID 115303, 2004.

[10] A. Matulis, F. M. Peeters, and P. Vasilopoulos, "Wave-vectordependent tunneling through magnetic barriers," Physical Review Letters, vol. 72, no. 10, pp. 1518-1521, 1994.

[11] V. Kubrak, F. Rahman, B. L. Gallagher et al., "Magnetoresistance of a two-dimensional electron gas due to a single magnetic barrier and its use for nanomagnetometry," Applied Physics Letters, vol. 74, no. 17, pp. 2507-2509, 1999.

[12] T. Vančura and M. Bichler, "Electron transport in a twodimensional electron gas with magnetic barriers," Physical Review B, vol. 62, no. 8, pp. 5074-5078, 2000.

[13] F. Zhai, H. Q. Xu, and Y. Guo, "Tunable spin polarization in a two-dimensional electron gas modulated by a ferromagnetic metal stripe and a Schottky metal stripe," Physical Review B, vol. 70, no. 8, Article ID 085308, 2004.

[14] G. Papp and F. M. Peeters, "Spin filtering in a magnetic-electric barrier structure," Applied Physics Letters, vol. 78, no. 15, pp. 2184-2186, 2001, Erratum, vol. 79, p. 3198, 2001.

[15] M.-W. Lu, X. H. Yan, and L. D. Zhang, "Spin polarization of electrons tunneling through magnetic-barrier nanostructures," Physical Review B, vol. 66, no. 22, Article ID 224412, 2002.

[16] M. Buttiker, "Four-terminal phase-coherent conductance," Physical Review Letters, vol. 57, no. 14, pp. 1761-1764, 1986. 

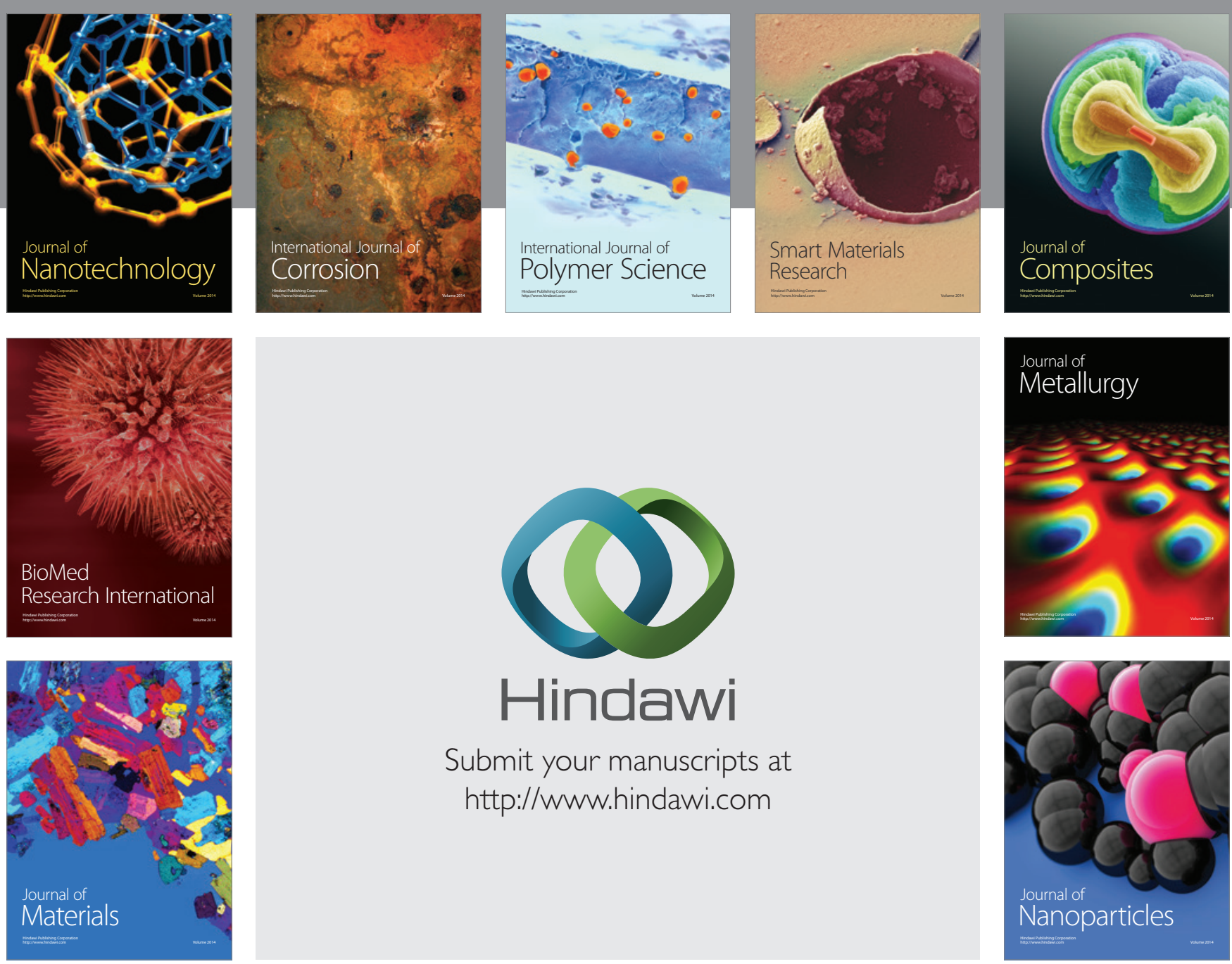

Submit your manuscripts at http://www.hindawi.com
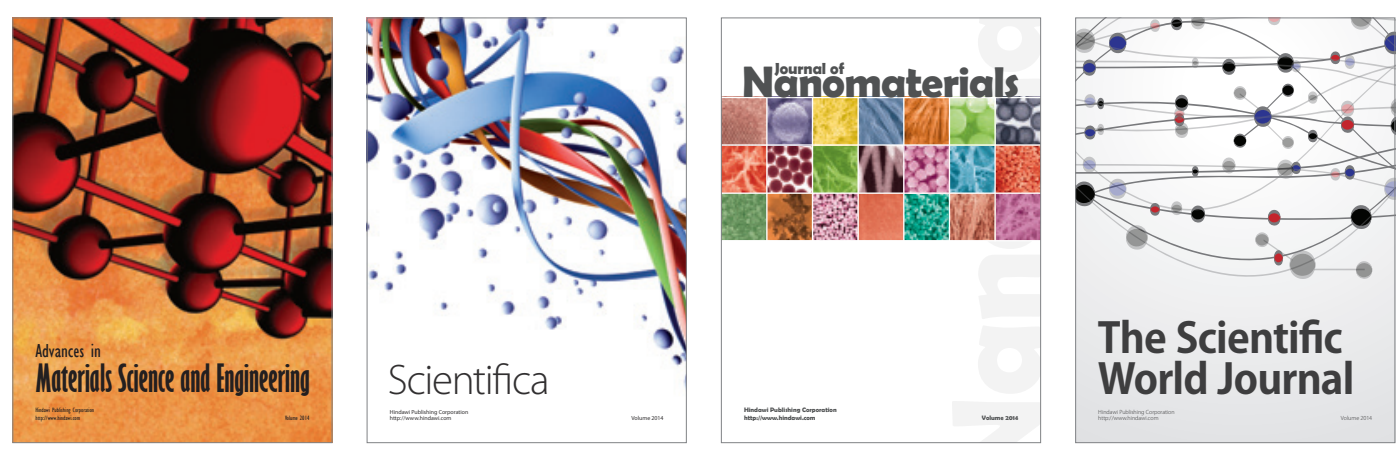

\section{The Scientific World Journal}
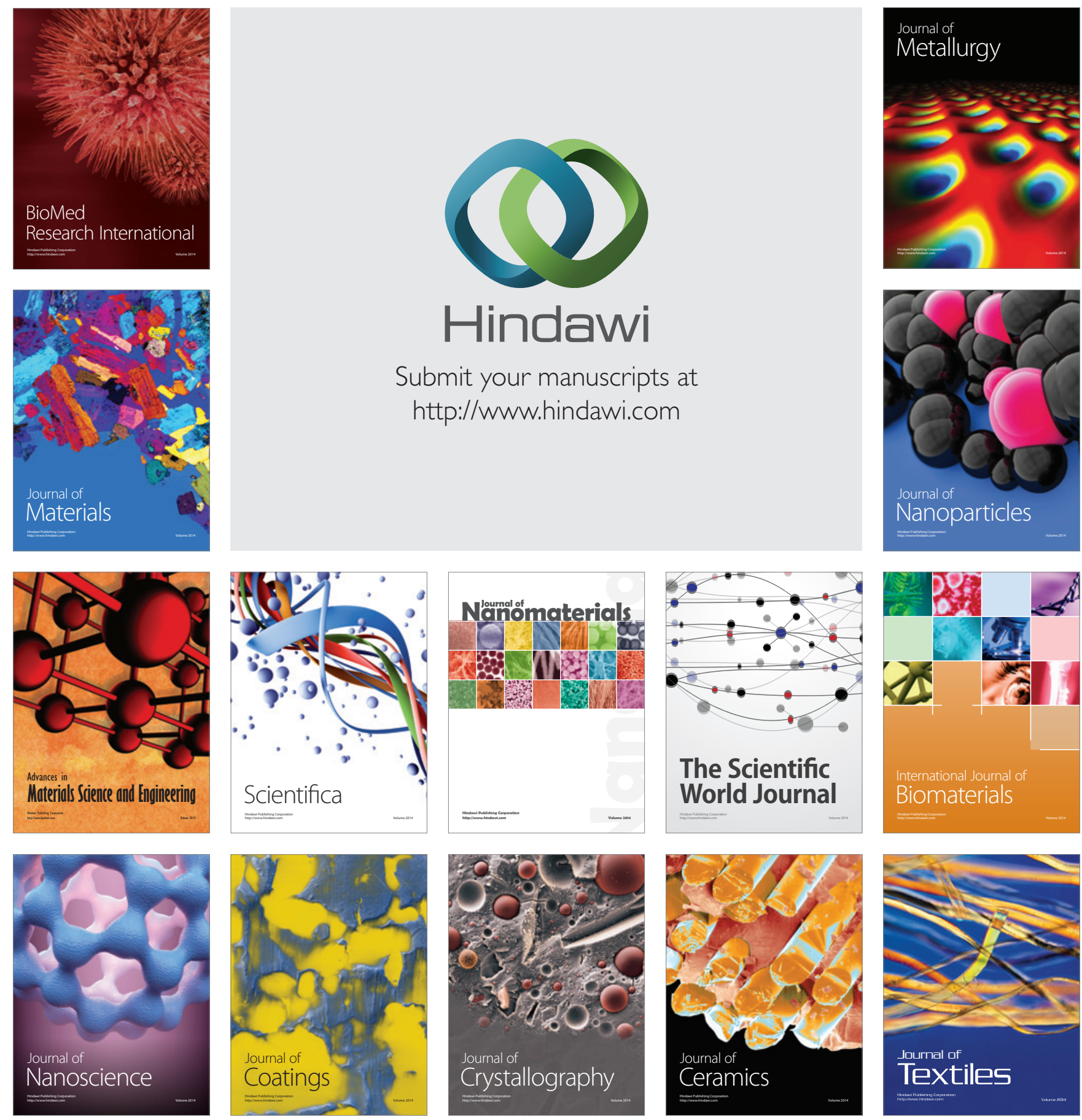